\title{
Lipid-Oligonucleotide Conjugates Forming G-Quadruplexes (Lipoquads) as Potent Inhibitors of HIV Entry ${ }^{\dagger}$
}

\author{
Sébastien Lyonnais ${ }^{1,2}$, Santiago Grijalvo ${ }^{3}$, Carmen Alvarez-Fernández ${ }^{1}$, Eric Fleta ${ }^{4}$, \\ Javier Martínez ${ }^{4}$, Andreas Meyerhans ${ }^{4}$, Sonsoles Sánchez-Palomino ${ }^{1}$, Gilles Mirambeau ${ }^{1}$ and \\ Ramon Eritja ${ }^{3, *}$
}

1 AIDS Research Group, IDIBAPS, 08036 Barcelona, Spain; seb.lyonnais@gmail.com or sebastien.lyonnais@cemipai.cnrs.fr (S.L.); caalvare@clinic.ub.es (C.A.-F.); ssanchez@clinic.ub.es (S.S.-P.); gilles.mirambeau@gmail.com (G.M.)

2 CEMIPAI, CNRS-UMS 3725, 3400 Montpellier, France

3 IQAC-CSIC, CIBER-BBN, 08034 Barcelona, Spain; santiago.grijalvo@iqac.csic.es

4 Infection Biology Laboratory, DCEXS, Universitat Pompeu Fabra, 08003 Barcelona, Spain; eric.fleta@upf.edu (E.F.); javier.martinez@upf.edu (J.M.); andreas.meyerhans@upf.edu (A.M.)

* Correspondence: ramon.eritja@iqac.csic.es

+ Presented at the 1st Molecules Medicinal Chemistry Symposium, Barcelona, Spain, 8 September 2017.

Published: 19 October 2017

Molecules that block virus entry by interfering with the actions of viral fusion proteins are of primary concern in the search for antiviral drugs. We present here the synthesis and antiviral activities of lipid-oligonucleotide conjugates (Lipoquads) forming a highly stable tetramolecular parallel G-quadruplex. We show that these molecules block HIV-1 and HIV-2 entry with submicromolar activities, demonstrating the great advantage of targeting both viral envelope glycoprotein and lipid rafts-a key platform in virus entry. Because the behavior of envelope proteins is similar in several other enveloped viruses, Lipoquads may have broader activities against enveloped viruses.

Supplementary Materials: The following are available online at www.mdpi.com/2504-3900/1/6/670/s: Poster S: Lipid-Oligonucleotide Conjugates Forming G-Quadruplex (Lipoquads) as Potent Inhibitors of HIV Entry.

Acknowledgments: Financial support by the Red de Investigación en Sida del Fondo de Investigación Sanitaria de la Seguridad Social (FIS) and the Spanish Ministry of Economy (MICINN) and Competitiveness and FEDER (Grants no. BFU2013-44629-R, CTQ2014-52588-R and SAF2013-46077-R). CIBER-BBN is an initiative funded by the VI National R+D+i Plan 2008-2011, Iniciativa Ingenio 2010, Consolider Program, CIBER Actions and financed by the Instituto de Salud Carlos III with assistance from the European Regional Development Fund. SL acknowledges funding by the Marie-Curie IEF fellowship (FP7-Grant Agreement 237738) and dedicates this work to the memory of Ian Fraser Kilmister.

Author Contributions: S.L., S.G., G.M., and R.E. designed, synthesized and purified the lipid-oligonucleotide conjugates as well as performed the assembly of the lipoquads. C.A.-F., S.S.-P., E.F., J.M., and A.M. characterized the antiviral properties of lipoquads.

Conflicts of Interest: The authors declare no conflicts of interest

(C) 2017 by the authors. Licensee MDPI, Basel, Switzerland. This article is an open access article distributed under the terms and conditions of the Creative Commons Attribution (CC BY) license (http://creativecommons.org/licenses/by/4.0/). 